\title{
THE EFFECT OF ILLICIT DRUGS ON ARTERIAL STIFFNESS IN YOUNG AND MIDDLE-AGED ILLICIT DRUG USERS: A POPULATION-BASED STUDY IN CHINA
}

\author{
Peng, X. ${ }^{1}-$ Zheng, L. ${ }^{1}{ }^{*}-$ Zhou, Z. ${ }^{3}-$ TAng, C. ${ }^{1}-$ JiAn, X. ${ }^{1}-$ Chen, Z. ${ }^{1}-$ TAN, J. ${ }^{1}-$ GuO, Y. ${ }^{1}$ \\ - LU, C. ${ }^{1}-$ ZhU, P. ${ }^{3}-\mathrm{LI}, \mathrm{J} .{ }^{1}-$ Cheng, D. ${ }^{1}-$ JiAng, Y. ${ }^{1}-$ WANG, W. ${ }^{1}-$ PENG, W. ${ }^{1}-$ SU, T. ${ }^{1}-$ \\ WANG, J. ${ }^{1}-\mathrm{LI}, \mathrm{H} .{ }^{1}-\mathrm{LIU}, \mathrm{Y}^{1}{ }^{1}-\mathrm{LUO}, \mathrm{J}^{1}{ }^{1}-\mathrm{WAN}, \mathrm{S} .{ }^{2 *}-\mathrm{ZHANG}, \mathrm{J}^{2 *}$ \\ ${ }^{1}$ Key Laboratory of Physical Fitness and Exercise Rehabilitation of Hunan Province, Hunan \\ Normal University, Chang Sha 410012, Hunan, China \\ ${ }^{2}$ Hunan Judicial Police Vocational College, Chang Sha 410126, Hunan, China \\ ${ }^{3}$ Guangdong Cardiovascular Institute, Guangdong General Hospital, Guangdong Academy of \\ Medical Sciences, Guangzhou 510100, Guangdong, China \\ *Corresponding authors \\ e-mail: Lanzheng@hunnu.edu.cn; wsp506@163.com; zhangju888aaa@163.com
}

(Received $25^{\text {th }}$ Sep 2019; accepted $11^{\text {th }}$ Nov 2019)

\begin{abstract}
Many illicit drugs have been linked to cardiovascular diseases, but large epidemiological studies are still needed to unveil the full effect of their use on the cardiovascular system. The aim of this study was to explore the relationship between illicit drug use and the prevalence of abnormal arterial stiffness in young and middle-aged illicit drug users. In total, 1247 illicit drug users and 107 non-illicit drug users were recruited for the Cardiovascular Damage Study, and 731 illicit drug users and 55 nonillicit drug users completed the cross-sectional analysis. Data was collected using questionnaires and a noninvasive vascular screening device. The value of brachial-ankle pulse wave velocity (baPWV) varied in a statistically significant manner depending on the type and duration of illicit drug use. The prevalence of abnormal baPWV varied significantly among different illicit drug user groups $\left(\chi^{2}=10.69, p<0.01\right)$. Multivariate logistic regression analysis revealed that age, sex, and the type of illicit drug used were crucial risk factors for abnormal baPWV. Older male narcotic drug users were associated with a significantly higher prevalence of abnormal baPWV. The main risk factors of arterial stiffness in young and middle-aged drug users were age, sex, and the type of illicit drug used.
\end{abstract}

Keywords: vascular elasticity, brachial-ankle pulse wave velocity, cardiovascular disease, narcotic drug, psychotropic drug, risk factor

\section{Introduction}

Abuse of illicit drugs results in a variety of pathophysiological changes to the cardiovascular system, such as the constriction of coronary vessels, dysfunction of the vascular endothelium, and decreased aortic elasticity. The cardiovascular impact of illicit drug use can lead to death, which is a growing public concern as the consumption of illicit drugs is increasing annually (Devlin and Henry, 2008; Maceira et al., 2014; Akkina et al., 2012). For example, cocaine abuse affects the elastic properties of the aorta and leads to increased systolic blood pressure and aortic stiffness (Bigi et al., 2008; Zhu et al., 2018). Other illicit drugs, including cannabis, amphetamines, and 3-4 methylenedioxymethamphetamine (MDMA), are also reported to be associated with hypertension, arrhythmia, and sudden death (Kaye et al., 2008; Heal et al., 2013; Singla et al., 2012; Wolff et al., 2011; Satoh et al., 2009). 
A means of early detection of cardiovascular system damage in illicit drug users would be significantly helpful for clinical and emergency treatment. Currently, an easily obtained, reproducible, and non-invasive parameter, known as brachial-ankle pulse wave velocity (baPWV), is considered to be a useful tool for the management of cardiovascular disease and its risk factors in illicit drug users. BaPWV mainly reflects segmental arterial elasticity (Kim et al., 2015). Since an increase in arterial stiffness will increase the propagation speed of a pulse wave in the artery (Tomiyama et al., 2016), baPWV can serve as a marker of arterial stiffness. A baPWV more than or equal to $1400 \mathrm{~cm} / \mathrm{s}$ indicates severe arterial stiffness (Yamashina et al., 2003; Wang et al., 2014) and a strong and independent association with coronary artery calcium (CAC), which is a powerful marker of coronary atherosclerosis (Cainzos-Achirica et al., 2015).

The cardiotoxic properties of illicit drugs have been studied for decades. Reports of the actual effects of illicit drugs on the cardiovascular systems of illicit drug users have not been well documented. Furthermore, the relationship between the abuse of various illicit drugs and arterial stiffness has not been reported.

According to the Illicit Drug Classification of United Nations Convention, illicit drugs were divided into two categories based on their pharmacological properties: narcotic and psychotropic. In this study, users of illicit narcotic drugs (opium, heroin, morphine, dolantin, marijuana, and cocaine) and illicit psychotropic drugs (methamphetamine, ketamine, and MDMA) were enrolled in the analysis. Users were divided into three groups: illicit narcotic drug users, illicit psychotropic drug users and users of both narcotic and psychotropic drugs (poly drug users). The possible relationships between the type, duration, and method of illicit drug abuse and arterial stiffness were evaluated using baPWV. The results of this study may provide new clues for the prediction and prevention of illicit drug abuse-related cardiovascular disease.

\section{Materials and methods}

\section{Subjects}

The illicit drug users were recruited from the drug rehabilitation centers of Hunan, Yunnan, Guangxi, Shanxi, and Guizhou provinces of China. The duration of survey was eight months which is from August 2017 to March 2018. A total of 1247 illicit drug users agreed to participate in the Cardiovascular Damage Study and signed the informed consent forms approved by the Institutional Ethics Committee of Hunan Normal University describing the procedures and assessments of the study. Subsequently, 878 cases completed the data collection. According to the results of the physical examination that participants completed when entering the drug rehabilitation center, 147 users did not meet the following selection criteria: (1) no history of metabolic diseases and organic diseases; (2) no cardiovascular drug treatment in the past five years; and $(3) \leq 45$ years of age. As such, 731 cases were enrolled in the final analysis. Non-illicit drug users were recruited from the local communities of Hunan, Yunnan, Guangxi, Shanxi, and Guizhou provinces of China. 107 non-illicit drug users agreed to participate and signed the informed consent forms of Cardiovascular Damage Study. After screening, 55 non-illicit drug users met the above mentioned selection criteria and were enrolled in the final analysis. 


\section{Questionnaires and quality control}

The questionnaires assessed demographic data and illicit drug abuse history including the type, duration, and method of illicit drug abuse. Participants were interviewed face-to-face by investigators trained to complete the questionnaire. All responses were reviewed by other trained investigators at the time of the interview.

\section{Measurements}

Blood pressure, baPWV and ankle-brachial index were measured using a BP-203 RPE III networked noninvasive vascular screening device produced by OMRON according to the manufacturer's instructions. The examination process was the same as previously descried (Wang et al., 2016). The subject was asked to sit for at least 5 min in an air-conditioned room $\left(22-25{ }^{\circ} \mathrm{C}\right)$. Participants wore light clothing and were instructed to lay in a supine position. Cuffs were placed on both arms and ankles and participants were asked to keep quiet during the examination process. All technicians were well-trained and familiar with the device. The measurements were performed by a single technician and were monitored by another researcher throughout the duration of the study.

\section{Statistical methods}

Statistical analysis was performed using R software, version 3.3.1 (R Core Team, 2016), and SPSS 20 (SPSS, Chicago, IL, USA). Continuous variables were presented as means \pm standard deviations (SD) and categorical variables were presented as percentages $(\%)$. For multiple comparisons of means, a pairwise t-test was used and $\mathrm{p}$ values were adjusted by false discovery rate (FDR) using the Benjaminni-Hochberg (BH) method. ANOVA was used to compare non-paired samples of normally distributed parameters. Pearson's chi-square test was used to compare prevalence differences. The aforementioned functions we used for data analysis were included in the $\mathrm{R}$ base package (R Core Team, 2016R Foundation, Australia). baPWV multivariate unconditional logistic regression analysis was analysed by both $\mathrm{R}$ base package and SPSS 20 . A p value less than 0.05 was considered statistically significant.

\section{Results}

\section{General characteristics of study illicit drug users}

In the end, 731 illicit drug users with complete personal information who fulfilled the selection criteria were included in the final analysis. The general characteristics of the subjects are presented in Table 1. Among the 731 illicit drug users, 535 (73.2\%) were male and $196(26.8 \%)$ were female. The mean age was 36.06 \pm 4.52 years (range 2345 years old). A total of 374 cases $(51.2 \%), 283$ cases $(38.7 \%)$, and 74 cases $(10.1 \%)$ were narcotic drug users, psychotropic drug users, and ploy drug users, respectively. Age, height, blood pressure, baPWV, and other indicators were significantly different among different illicit drug users (Table 1).

Statistical analysis revealed that baPWV exhibited significant differences based on the type and the duration of illicit drug used (Tables 1 and 2). No significant difference was observed in baPWV value based on the method of illicit drug abuse (Table Al in the Appendix). 
Table 1. Baseline characteristics of the study subjects

\begin{tabular}{c|c|c|c}
\hline & Narcotic $(\mathbf{n}=\mathbf{3 7 4})$ & Psychotropic $(\mathbf{n}=\mathbf{2 8 3})$ & Poly $(\mathbf{n}=\mathbf{7 4})$ \\
\hline Age, years & $37.45 \pm 4.11$ & $34.41 \pm 4.54^{\mathrm{a}}$ & $35.36 \pm 3.96^{\mathrm{a}}$ \\
Height, cm & $163.19 \pm 7.73$ & $166.93 \pm 7.63^{\mathrm{a}}$ & $163.81 \pm 7.78^{\mathrm{b}}$ \\
Weight, Kg & $61.01 \pm 9.88$ & $64.56 \pm 10.19^{\mathrm{a}}$ & $60.71 \pm 11.04^{\mathrm{b}}$ \\
BMI, Kg/m ${ }^{2}$ & $22.90 \pm 3.30$ & $23.17 \pm 3.24$ & $22.56 \pm 3.10$ \\
Vital capacity, ml & $2674.70 \pm 692.28$ & $2881.44 \pm 747.41^{\mathrm{a}}$ & $2669.22 \pm 697.39^{\mathrm{b}}$ \\
Heart rate, b/min & $78.52 \pm 13.22$ & $75.04 \pm 11.70^{\mathrm{a}}$ & $75.74 \pm 10.72$ \\
SBP, mmHg & $119.51 \pm 15.86$ & $118.84 \pm 29.22$ & $116.81 \pm 15.44$ \\
DBP, mmHg & $75.88 \pm 11.35$ & $71.98 \pm 11.70^{\mathrm{a}}$ & $75.74 \pm 10.72^{\mathrm{a}}$ \\
RbaPWV & $1226.82 \pm 186.09$ & $1178.67 \pm 126.18^{\mathrm{a}}$ & $1176.74 \pm 155.33^{\mathrm{a}}$ \\
LbaPWV & $1227.59 \pm 178.66$ & $1186.52 \pm 122.04^{\mathrm{a}}$ & $1184.35 \pm 153.33$ \\
Rabi & $1.07 \pm 0.10$ & $1.07 \pm 0.10^{\mathrm{a}}$ & $1.05 \pm 0.08$ \\
Labi & $1.06 \pm 0.10$ & $1.07 \pm 0.10^{\mathrm{a}}$ & $1.06 \pm 0.08$ \\
IAD, mmHg & $3.63 \pm 3.22$ & $3.62 \pm 3.24$ & $2.81 \pm 2.50$ \\
Duration, months & $71.32 \pm 80.76$ & $50.47 \pm 47.47^{\mathrm{a}}$ & $94.68 \pm 70.51^{\mathrm{ab}}$ \\
\hline
\end{tabular}

BMI: body mass index; SBP: systolic blood pressure; DBP: diastolic blood pressure; Rabi: right anklebrachial index; Labi: left ankle-brachial index; RbaPWV: right brachial-ankle pulse wave velocity; LbaPWV: left brachial-ankle pulse wave velocity; IAD: inter-arm blood pressure difference. The $p$ values were adjusted by false discovery rate (FDR) using the Benjamini-Hochberg (BH) method. ${ }^{\mathrm{a}} p<0.05$ versus the narcotic drug users; ${ }^{\mathrm{b}} p<0.05$ versus the psychotropic drug users

Table 2. Comparison of the baPWV value among the different illicit drug abuse duration groups

\begin{tabular}{c|c|c}
\hline Duration, months & RbaPWV & LbaPWV \\
\hline 12 & $1241.13 \pm 209.38$ & $1246.89 \pm 197.42$ \\
36 & $1202.73 \pm 165.19$ & $1206.44 \pm 152.74$ \\
72 & $1186.49 \pm 167.59$ & $1196.92 \pm 158.71$ \\
144 & $1183.97 \pm 166.19$ & $1187.82 \pm 159.04$ \\
$>144$ & $1181.63 \pm 146.48$ & $1175.40 \pm 156.52$ \\
$p$ value & 0.04 & $<0.01$ \\
\hline
\end{tabular}

RbaPWV: right brachial-ankle pulse wave velocity. One-way ANOVA was used to analyze the difference among different drug abuse duration groups

\section{Analysis of the prevalence of abnormal baPWV in different sex, age, and drug user groups}

The total detection rates of abnormal baPWV in illicit drug users (14.4\%) were significantly greater than those of non-illicit drug users $(3.6 \%)\left(\chi^{2}=4.13, p<0.05\right)$ (Table 3). To investigate the risk factors for abnormal baPWV in illicit drug users, the prevalence of abnormal baPWV in different sex, age, and drug user groups were analyzed. The results showed that the prevalence of abnormal baPWV was not significantly different between male and female illicit drug users $\left(\chi^{2}=3.76, p>0.05\right)$ (Table A2 in the Appendix). It has been reported that peripheral vascular resistance increases and arterial elasticity decreases with age, which results in a higher prevalence of abnormal baPWV in the elderly population (Ishida et al., 2018). Therefore, according 
to the age composition of the illicit drug users, the cases were divided into three groups. The detection rate of abnormal baPWV in illicit drug users between the ages of 21-30, $31-40$, and over 40 was $8.0 \%, 14.3 \%$, and $19.3 \%$, respectively. There was no significant difference in the prevalence of abnormal baPWV among different age groups $\left(\chi^{2}=5.32\right.$, $\mathrm{p}>0.05)$ (Table A3).

Table 3. Comparison of abnormal baPWV prevalence rates between illicit drug users and non-illicit drug users

\begin{tabular}{c|c|c|c}
\hline & baPWV $\geq \mathbf{1 4 0 0}$ & baPWV $<\mathbf{1 4 0 0}$ & Total \\
\hline Illicit drug users & $626(85.6 \%)$ & $105(14.4 \%)$ & 731 \\
Non-illicit drug users & $2(3.6 \%)$ & $53(96.4 \%)$ & 55 \\
\hline
\end{tabular}

baPWV: brachial-ankle pulse wave velocity. The difference between each group was analyzed by Pearson's chi-square test $\chi^{2}=4.13, p<0.05$

Since the value of baPWV showed significant differences among different illicit drug abuse duration groups, the prevalence of abnormal baPWV differences among them was further analyzed. The detection rate of abnormal baPWV in different illicit drug abuse duration groups of $0-12,13-36,37-72,73-144$ and over 144 months was $20.1 \%$, $14.4 \%, 13.0 \%, 11.3 \%$, and $9.4 \%$, respectively, but no significant difference was found among these groups $\left(\chi^{2}=7.90, \mathrm{p}>0.05\right)$ (Table A4). Nevertheless, our further investigation of cardiovascular damage in illicit drug users based on baPWV revealed that the prevalence of abnormal baPWV showed a significant difference between the different illicit drug user groups: $18.4 \%$ in the narcotic drug user group, $10.6 \%$ in the psychotropic drug user group, and $8.1 \%$ in the poly drug user group $\left(\chi^{2}=10.69\right.$, $\mathrm{p}<0.01)($ Table 4).

Table 4. Comparison of abnormal baPWV prevalence rates of different type illicit drug users

\begin{tabular}{c|c|c|c}
\hline & baPWV $\geq \mathbf{1 4 0 0}$ & baPWV < 1400 & Total \\
\hline Narcotic & $69(18.4 \%)$ & $305(81.6 \%)$ & $374(51.2 \%)$ \\
Psychotropic & $30(10.6 \%)$ & $253(89.4 \%)$ & $283(38.7 \%)$ \\
Poly & $6(8.1 \%)$ & $68(91.9 \%)$ & $74(10.1 \%)$ \\
Total & $105(14.4 \%)$ & $626(85.6 \%)$ & 731 \\
\hline
\end{tabular}

baPWV: brachial-ankle pulse wave velocity. The difference between each group was analyzed by Pearson's chi-square test $\chi^{2}=10.69, p<0.01$

\section{Analysis of the relevant risk factors for abnormal baPWV in illicit drug users}

To avoid possible interactions between each risk factor, multivariate logistic regression analysis was used to evaluate the effect of illicit drug abuse on arterial stiffness. The result showed that significant predictors of abnormal baPWV were age, sex, and the type of illicit drug used. In males, the risk of abnormal baPWV was 1.97fold higher $(\mathrm{OR}=1.97,95 \% \mathrm{CI}=1.11-3.47)$ than that in females. The risk of developing abnormal baPWV increased by 1.10 -fold per year of age $(\mathrm{OR}=1.10,95 \%$ $\mathrm{CI}=1.04-1.17)$. Compared with narcotic drug users $(\mathrm{OR}=1)$, the risk of abnormal baPWV in psychotropic drug users $(\mathrm{OR}=0.49,95 \% \mathrm{CI}=0.28-0.84)$ and poly drug users $(\mathrm{OR}=0.63,95 \% \mathrm{CI}=0.23-1.72)$ was lower (Table 5). 
Table 5. Multivariate unconditional logistic regression analysis of risk factors for abnormal baPWV in illicit drug users

\begin{tabular}{c|c|c|c|c|c|c|c}
\hline & B & S. E. & Wald & df & $\boldsymbol{p}$ value & OR & 95\% CI \\
\hline Sex & 0.68 & 0.29 & 5.42 & 1 & 0.02 & 1.97 & $1.11-3.47$ \\
Age & 0.10 & 0.03 & 3.52 & 1 & $<0.01$ & 1.10 & $1.04-1.17$ \\
BMI & & & 1.71 & 3 & 0.64 & & \\
normal & & & & & & 1 & \\
Slim & 0.43 & 0.68 & 0.39 & 1 & 0.53 & 1.53 & $0.40-5.82$ \\
Overweight & 0.11 & 0.27 & 0.16 & 1 & 0.69 & 1.12 & $0.65-1.90$ \\
Obesity & 0.59 & 0.50 & 1.36 & 1 & 0.24 & 1.80 & $0.67-4.82$ \\
Drug type & & & 6.91 & 2 & 0.03 & & \\
narcotic & & & & & & 1 & \\
Psychotropic & -0.72 & 0.28 & 6.65 & 1 & 0.01 & 0.49 & $0.28-0.84$ \\
Poly & -0.46 & 0.51 & 0.81 & 1 & 0.37 & 0.63 & $0.23-1.72$ \\
Duration & & & 5.66 & 4 & 0.23 & & \\
0-12 m & & & & & & 1 & \\
13-36 m & -0.21 & 0.30 & 0.51 & 1 & 0.48 & 0.81 & $0.45-1.45$ \\
37-72 m & -0.39 & 0.35 & 1.23 & 1 & 0.27 & 0.68 & $0.34-1.35$ \\
73-144 m & -0.64 & 0.39 & 2.72 & 1 & 0.10 & 0.53 & $0.25-1.13$ \\
$>144 \mathrm{~m}$ & -0.85 & 0.42 & 4.11 & 1 & 0.04 & 0.43 & $0.19-0.97$ \\
Method & & & 1.73 & 6 & 0.94 & & \\
sucking & & & & & & 1 & \\
P.O & -0.17 & 0.53 & 0.11 & 1 & 0.74 & 0.84 & $0.30-2.36$ \\
Snorting & -15.04 & 686.82 & 0.00 & 1 & 1.00 & 0.00 & 0.00 \\
IV & -0.29 & 0.34 & 0.72 & 1 & 0.40 & 0.75 & $0.39-1.46$ \\
Smoking & -0.44 & 0.47 & 0.87 & 1 & 0.35 & 0.65 & $0.26-1.62$ \\
mixture & 0.22 & 0.61 & 0.13 & 1 & 0.72 & 1.24 & $0.38-4.09$ \\
others & -0.24 & 1.11 & 0.05 & 1 & 0.83 & 0.79 & $0.09-6.91$ \\
Constant & -5.26 & 1.16 & 20.56 & 1 & 0.00 & 0.01 & \\
\hline P.O. per & & & & \\
\hline
\end{tabular}

P.O: per os, IV: intravenous injection

A predictive model of the risk of arterial stiffness of illicit drug users (ASIDU) was developed based on multivariate logistic regression analysis according to the value of baPWV collected in this study. This ASIDU model contains six factors, including sex, age, Body Mass Index (BMI), the type of illicit drug used, the duration of illicit drug used and the method of taking illicit drugs. To evaluate the predictive performance of the ASIDU model, Receiver Operating Characteristic (ROC) analysis was used. The ASIDU model exhibited an area under the ROC curve (AUC) of $0.70(95 \% \mathrm{CI}=0.64-0.75)$. The cut-off point (male, 29 years old, overweight, narcotic drug users and 12-36 months of illicit drug abuse) showed $60 \%$ sensitivity and $72 \%$ specificity in predicting arterial stiffness of illicit drug users according to the value of baPWV (Fig. 1).

\section{Discussion}

The present study indicates that cardiovascular damage in illicit drug users is significantly associated with age, sex, and the type of illicit drug used. Among the 731 
young and middle-aged illicit drug users in our study, 105 cases (14.4\%) exhibited various degrees of abnormal arterial stiffness. It is significantly higher than the detection rate of that in the young and middle-aged normal population (3.6\%). This indicates that abnormal arterial stiffness occurs in illicit drug users at a relatively young age. The incidence of cardiovascular disease and cerebral infarction induced by arterial stiffness is increasing year by year (Mikael et al., 2017; Hatanaka et al., 2011). Thus, detection of the changes in arterial stiffness in high-risk drug users can effectively predict the occurrence and development of cardiovascular and cerebrovascular diseases, which may reduce mortality among illicit drug users.

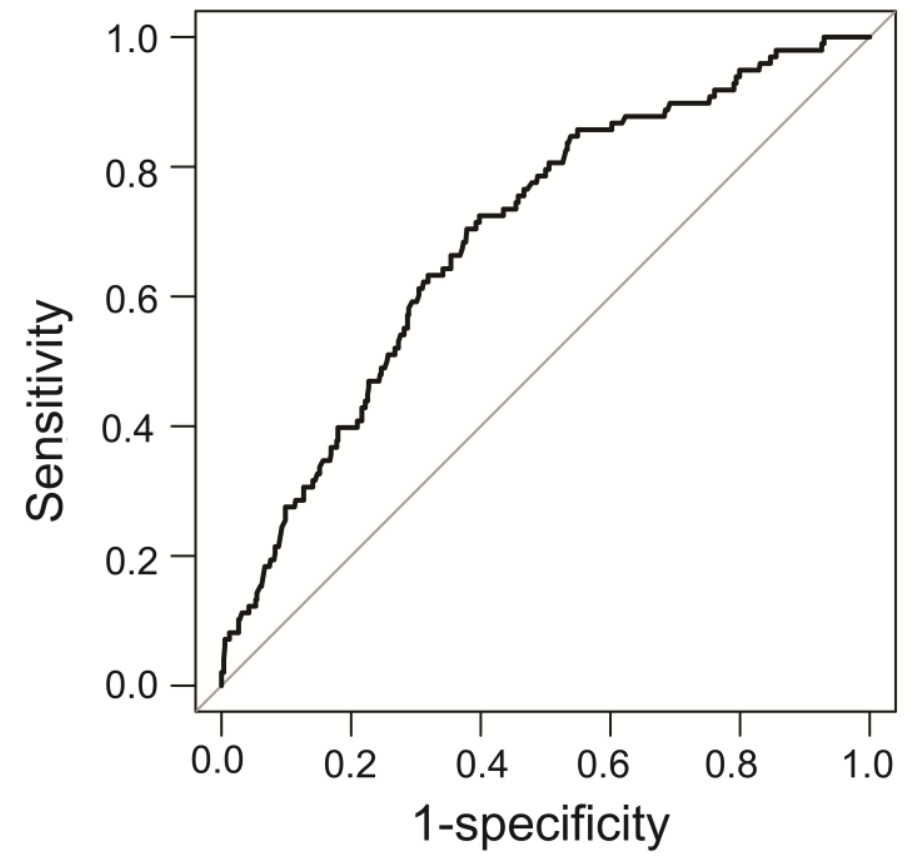

Figure 1. ROC curve analysis of the predictive performance of the ASIDU model. AUC $=0.70$, 95\% CI = 0.64-0.75. (ROC: Receiver operating characteristic; ASIDU: arterial stiffness of illicit drug users; AUC: area under curve)

Previous reports demonstrated that age is a major determinant of arterial stiffness (Lee and Oh, 2010). During the aging process, the vessel wall undergoes a variety of changes, including increased calcium deposition, quantitative and qualitative changes in medial layer collagen, and arterial wall hypertrophy. These results in disorganized microarchitecture, decreased vessel wall compliance and elasticity, and increased arterial stiffness (Tomiyama et al., 2003). Individuals more than 45 years old are more susceptible to arterial stiffness (Ishida et al., 2018). In addition, metabolic problems and a sedentary lifestyle also are critical factors affecting the arterial stiffness process (Satoh et al., 2009; Garcia-Hermoso et al., 2015). Thus, individuals less than 45 years old without metabolic and organic diseases were recruited to better evaluate the effect of illicit drugs on the cardiovascular system.

Some illicit drugs have been found to be associated with cardiovascular problems, such as arrhythmia, hypertension, myocardial infarction (MI), and atherosclerosis. In the United States, cocaine is the cause of $25 \%$ of MIs in people under 45 years of age (Qureshi et al., 2001). Up to $83 \%$ of asymptomatic cocaine users suffer from silent 
myocardial injuries such as myocardial edema and myocardial fibrosis (Aquaro et al., 2011). Cardiovascular pathology, typically atherosclerosis, has been detected in $58 \%$ of MDMA users. This detection rate is higher than that expected among similarly aged members of the general population (Kaye et al., 2009). Amphetamine and cannabis are also thought to be linked to various cardiovascular problems, such as hypertension, cardiac arrhythmia and increased heart rate (Vitiello, 2008). Although the cardiotoxic properties of some of these illicit drugs have been well documented theoretically, the exact effect of different types of illicit drugs on humans has not been fully investigated. Our study revealed that illicit narcotic drug users have a higher detection rate of abnormal baPWV than illicit psychotropic drug users, which indicates that illicit narcotic drugs have a stronger effect on cardiovascular damage than that of illicit psychotropic drugs.

In a study of 121 intravenous drug abusers, 95 had systolic murmurs and 44 had extra-cardiac infections (Hecht and Berger, 1992). Vitiello et al. found that the effect of illicit abuse via intravenous injection is more pronounced that that associated with other methods of illicit drug abuse (Vitiello, 2008). However, in this study, we did not find a significant difference between different methods of drug abuse. This discrepancy may be a result of differences in the characteristics of the studied populations. Equal sample selection criteria are required to make a parallel comparison between the results.

\section{Conclusion}

In summary, age, sex and type of illicit drug used were crucial risk factors for abnormal arterial stiffness in young and middle-aged illicit drug users. The effects of different durations of illicit drug abuse on the cardiovascular system were compared, but no significant difference was found after multivariate logistic regression analysis. This may be due to the fact that the effect of illicit drug abuse is generated by the accumulation of cardiotoxicity, which is not only determined by the duration of drug abuse but is also related to the frequency of drug abuse and the average amount of illicit drug used in each time. Therefore, in order to further explore the cardiovascular impact illicit drug use, the duration and frequency of drug abuse and the average amount of illicit drug used should be taken into consideration.

Acknowledgments. This research was supported by grants from National Natural Science Foundation of China (31671243, 81801392, 81570279), National Key Research and Development Program of China (2016YFC0800908, 2018YFA0108700), and China Postdoctoral Science Foundation (2018M630903).

\section{REFERENCES}

[1] Akkina, S. K., Ricardo, A. C., Patel, A., Das, A., Bazzano, L. A., Brecklin, C., Fischer, M. J., Lash, J. P. (2012): Illicit drug use, hypertension, and chronic kidney disease in the US adult population. - Translational Research: The Journal of Laboratory and Clinical Medicine 160(6): 391-398.

[2] Aquaro, G. D., Gabutti, A., Meini, M., Prontera, C., Pasanisi, E., Passino, C., Emdin, M., Lombardi, M. (2011): Silent myocardial damage in cocaine addicts. - Heart 97(24): 2056-2062.

[3] Bigi, M. A., Aslani, A., Mehrpour, M. (2008): Effect of chronic cocaine abuse on the elastic properties of aorta. - Echocardiography 25(3): 308-311. 
[4] Cainzos-Achirica, M., Rampal, S., Chang, Y., Ryu, S., Zhang, Y., Zhao, D., Cho, J., Choi, Y., Pastor-Barriuso, R., Lim, S. Y., Bruguera, J., Elosua, R., Lima, J. A., Shin, H., Guallar, E. (2015): Brachial-ankle pulse wave velocity is associated with coronary calcium in young and middle-aged asymptomatic adults: the Kangbuk Samsung Health Study. - Atherosclerosis 241(2): 350-356.

[5] Devlin, R. J., Henry, J. A. (2008): Clinical review: major consequences of illicit drug consumption. - Critical Care 12(1): 202.

[6] Garcia-Hermoso, A., Notario-Pacheco, B., Recio-Rodriguez, J. I., Martinez-Vizcaino, V., Rodrigo de Pablo, E., Magdalena Belio, J. F., Gomez-Marcos, M. A., Garcia-Ortiz, L., Group, E. (2015): Sedentary behaviour patterns and arterial stiffness in a Spanish adult population - the EVIDENT trial. - Atherosclerosis 243(2): 516-522.

[7] Hatanaka, R., Obara, T., Watabe, D., Ishikawa, T., Kondo, T., Ishikura, K., Aikawa, T., Aono, Y., Hara, A., Metoki, H., Asayama, K., Kikuya, M., Mano, N., Ohkubo, T., Izumi, S., Imai, Y. (2011): Association of arterial stiffness with silent cerebrovascular lesions: the Ohasama study. - Cerebrovascular Diseases 31(4): 329-337.

[8] Heal, D. J., Smith, S. L., Gosden, J., Nutt, D. J. (2013): Amphetamine, past and present a pharmacological and clinical perspective. - Journal of Psychopharmacology 27(6): 479496.

[9] Hecht, S. R., Berger, M. (1992): Right-sided endocarditis in intravenous drug users. Prognostic features in 102 episodes. - Annals of Internal Medicine 117(7): 560-566.

[10] Ishida, A., Fujisawa, M., Del Saz, E. G., Okumiya, K., Kimura, Y., Manuaba, I. I. B., Kareth, M. F., Rantetampang, A. L., Ohya, Y., Matsubayashi, K. (2018): Arterial stiffness, not systolic blood pressure, increases with age in native Papuan populations. Hypertension Research: Official Journal of the Japanese Society of Hypertension 41(7): 539-546.

[11] Kaye, S., Darke, S., Duflou, J., McKetin, R. (2008): Methamphetamine-related fatalities in Australia: demographics, circumstances, toxicology and major organ pathology. Addiction 103(8): 1353-1360.

[12] Kaye, S., Darke, S., Duflou, J. (2009): Methylenedioxymethamphetamine (MDMA)related fatalities in Australia: demographics, circumstances, toxicology and major organ pathology. - Drug and Alcohol Dependence 104(3): 254-261.

[13] Kim, E. K., Chang, S. A., Jang, S. Y., Choi, K. H., Huh, E. H., Kim, J. H., Kim, S. M., Choe, Y. H., Kim, D. K. (2015): Brachial-ankle pulse wave velocity as a screen for arterial stiffness: a comparison with cardiac magnetic resonance. - Yonsei Medical Journal 56(3): 617-624.

[14] Lee, H. Y., Oh, B. H. (2010): Aging and arterial stiffness. - Circulation Journal: Official Journal of the Japanese Circulation Society 74(11): 2257-2262.

[15] Maceira, A. M., Ripoll, C., Cosin-Sales, J., Igual, B., Gavilan, M., Salazar, J., Belloch, V., Pennell, D. J. (2014): Long term effects of cocaine on the heart assessed by cardiovascular magnetic resonance at 3T. - Journal of Cardiovascular Magnetic Resonance: Official Journal of the Society for Cardiovascular Magnetic Resonance 16: 26.

[16] Mikael, L. R., Paiva, A. M. G., Gomes, M. M., Sousa, A. L. L., Jardim, P., Vitorino, P. V. O., Euzebio, M. B., Sousa, W. M., Barroso, W. K. S. (2017): Vascular aging and arterial stiffness. - Arquivos Brasileiros de Cardiologia 109(3): 253-258.

[17] Qureshi, A. I., Suri, M. F., Guterman, L. R., Hopkins, L. N. (2001): Cocaine use and the likelihood of nonfatal myocardial infarction and stroke: data from the Third National Health and Nutrition Examination Survey. - Circulation 103(4): 502-506.

[18] R Core Team (2016): R: A Language and Environment for Statistical Computing. - R Foundation for Statistical Computing, Vienna, Austria.

[19] Satoh, H., Kishi, R., Tsutsui, H. (2009): Metabolic syndrome is a significant and independent risk factor for increased arterial stiffness in Japanese subjects. Hypertension Research: Official Journal of the Japanese Society of Hypertension 32(12): 1067-1071. 
[20] Singla, S., Sachdeva, R., Mehta, J. L. (2012): Cannabinoids and atherosclerotic coronary heart disease. - Clinical Cardiology 35(6): 329-335.

[21] Tomiyama, H., Yamashina, A., Arai, T., Hirose, K., Koji, Y., Chikamori, T., Hori, S., Yamamoto, Y., Doba, N., Hinohara, S. (2003): Influences of age and gender on results of noninvasive brachial-ankle pulse wave velocity measurement - a survey of 12517 subjects. - Atherosclerosis 166(2): 303-309.

[22] Tomiyama, H., Matsumoto, C., Shiina, K., Yamashina, A. (2016): Brachial-ankle PWV: current status and future directions as a useful marker in the management of cardiovascular disease and/or cardiovascular risk factors. - Journal of Atherosclerosis and Thrombosis 23(2): 128-146.

[23] Vitiello, B. (2008): Understanding the risk of using medications for attention deficit hyperactivity disorder with respect to physical growth and cardiovascular function. Child and Adolescent Psychiatric Clinics of North America 17(2): 459-474.

[24] Wang, A., Tao, J., Guo, X., Liu, X., Luo, Y., Liu, X., Huang, Z., Chen, S., Zhao, X., Jonas, J. B., Wu, S. (2014): The product of resting heart rate times blood pressure is associated with high brachial-ankle pulse wave velocity. - PloS One 9(9): 107852.

[25] Wang, Y., Yang, Y., Wang, A., An, S., Li, Z., Zhang, W., Liu, X., Ruan, C., Liu, X., Guo, X., Zhao X., Wu, S. (2016): Association of long-term blood pressure variability and brachial-ankle pulse wave velocity: a retrospective study from the APAC cohort. Scientific Reports 6: 21303.

[26] Wolff, V., Lauer, V., Rouyer, O., Sellal, F., Meyer, N., Raul, J. S., Sabourdy, C., Boujan, F., Jahn, C., Beaujeux, R., Marescaux, C. (2011): Cannabis use, ischemic stroke, and multifocal intracranial vasoconstriction: a prospective study in 48 consecutive young patients. - Stroke 42(6): 1778-1780.

[27] Yamashina, A., Tomiyama, H., Arai, T., Hirose, K., Koji, Y., Hirayama, Y., Yamamoto, Y., Hori, S. (2003): Brachial-ankle pulse wave velocity as a marker of atherosclerotic vascular damage and cardiovascular risk. - Hypertension Research: Official Journal of the Japanese Society of Hypertension 26(8): 615-622.

[28] Zhu, W., Wang, H., Wei, J., Sartor, G. C., Bao, M. M., Pierce, C. T., Wahlestedt, C. R., Dykxhoorn, D. M., Dong, C. (2018): Cocaine exposure increases blood pressure and aortic stiffness via the miR-30c-5p-malic enzyme 1-reactive oxygen species pathway. Hypertension 71(4): 752-760.

\section{APPENDIX}

Table A1. Comparison of the baPWV value among different drug abuse method groups

\begin{tabular}{c|c|c}
\hline Methods & RbaPWV & LbaPWV \\
\hline Sucking & $1200.61 \pm 179.05$ & $1209.56 \pm 173.91$ \\
P.O & $1231.21 \pm 140.65$ & $1234.88 \pm 132.89$ \\
Snorting & $1183.18 \pm 173.7$ & $1172.09 \pm 152.04$ \\
IV & $1210.49 \pm 187.06$ & $1203.13 \pm 170.34$ \\
Smoking & $1206.02 \pm 144.25$ & $1207.06 \pm 142.14$ \\
Mixture & $1259.86 \pm 221.81$ & $1253.33 \pm 187.26$ \\
others & $1150.00 \pm 170.59$ & $1156.00 \pm 175.33$ \\
$p$ value & 0.63 & 0.65 \\
\hline
\end{tabular}

RbaPWV: right brachial-ankle pulse wave velocity; LbaPWV: left brachial-ankle pulse wave velocity; P.O: per os; IV: intravenous injection. One-way ANOVA was used to analyze the difference among different drug abuse method groups 
Table A2. Comparison of abnormal baPWV prevalence rates between males and females

\begin{tabular}{c|c|c|c}
\hline & baPWV $\geq \mathbf{1 4 0 0}$ & baPWV < 1400 & Total \\
\hline Male & $85(16.9 \%)$ & $450(83.1 \%)$ & $535(73.2 \%)$ \\
Female & $20(10.2 \%)$ & $176(89.8 \%)$ & $196(26.8 \%)$ \\
Total & $105(14.4 \%)$ & $626(85.6 \%)$ & 731 \\
\hline
\end{tabular}

baPWV: brachial-ankle pulse wave velocity. The difference between each group was analyzed by Pearson's chi-square test $\chi^{2}=3.76, p>0.05$

Table A3. Comparison of abnormal baPWV prevalence rates among different age groups

\begin{tabular}{c|c|c|c}
\hline Age, years & baPWV $\geq \mathbf{1 4 0 0}$ & baPWV < 1400 & Total \\
\hline $21-30$ & $7(8.0 \%)$ & $81(92.0 \%)$ & $88(12.0 \%)$ \\
$31-40$ & $75(14.3 \%)$ & $449(85.7 \%)$ & $524(71.7 \%)$ \\
$>40$ & $23(19.3 \%)$ & $96(80.7 \%)$ & $119(16.3 \%)$ \\
Total & $105(14.4 \%)$ & $626(85.6 \%)$ & 731 \\
\hline
\end{tabular}

baPWV: brachial-ankle pulse wave velocity. The difference between each group was analyzed by Pearson's chi-square test $\chi^{2}=5.32, p>0.05$

Table A4. Comparison of abnormal baPWV prevalence rates of different type illicit drug abuse durations

\begin{tabular}{c|c|c|c}
\hline Duration, months & baPWV $\geq \mathbf{1 4 0 0}$ & baPWV < 1400 & Total \\
\hline $0-12$ & $36(20.1 \%)$ & $143(79.9 \%)$ & $179(24.5 \%)$ \\
$13-36$ & $28(14.4 \%)$ & $166(85.6 \%)$ & $194(26.5 \%)$ \\
$37-72$ & $18(13.0 \%)$ & $120(87.0 \%)$ & $138(18.9 \%)$ \\
$73-144$ & $14(11.3 \%)$ & $110(88.7 \%)$ & $124(17.0 \%)$ \\
$>144$ & $9(9.4 \%)$ & $87(80.6 \%)$ & $96(13.1 \%)$ \\
Total & $105(14.4 \%)$ & $626(85.6 \%)$ & 731 \\
\hline
\end{tabular}

baPWV: brachial-ankle pulse wave velocity. The difference between each group was analyzed by Pearson's chi-square test $\chi^{2}=7.90, p>0.05$ 